\section{Light from seismic waves}

SIR - Sonoluminescence (SL) - the production of light by the action of sound waves in liquid - has been observed and studied in the laboratory for more than 50 years. But I believe it has been observed in nature for centuries as earthquake lights (EQLs). EQLs second only to pre-seismic abnormal animal behaviour for difficulty of reliable documentation and lack of a verifiable explanatory mechanism.

Many explanations for the generation of EQLs exist (see refs 1-3). Most proposed mechanisms require assumptions such as the presence of special minerals, gases or organisms, or unverified physical conditions in the fault zone. All have difficulty explaining the persistent reports of EQLs at distances of up to hundreds of kilometres from the earthquake, and at sea or in association with large bodies of both fresh and salt water or wetlands.

I propose that at least some EQLs are not the product of high strain accumulation or shear rupture dynamics in fault zones, but rather result from molecular reactions in water that has been strongly shaken by the compressional $(P)$ waves produced by the earthquake. A seismic $P$ wave is simply an earthquakegenerated sound wave in a solid or liquid; hence if a $P$ wave induces light emission from liquid, it is a situation entirely analogous to SL as generated in the chemist's laboratory.

$\mathrm{SL}$ is a remarkable consequence of acoustic cavitation in liquids irradiated by sound waves ${ }^{4,5}$. For it to occur, a cavity or bubble must be created in the liquid continuum, then rapidly compressed. The process adiabatically heats the trapped gas or vapour sufficiently to dissociate molecules. On recombination or return to the ground state, photons are emitted. Once a cavity or bubble is formed, two types of SL are possible: 'stable', in which the bubble resonates and incrementally grows, usually in a standing wave field; and 'transient', in which the bubble expands and implodes all within one cycle of a standing or travelling sound wave. A travelling $P$ wave should be an efficient stimulus of transient cavitation, although long trains or strong-motion $P$ waves may induce stable cavitation SL as well.

The observed SL spectrum in water has a peak at $310 \mathrm{~nm}$ (in the ultraviolet), arising from the return to the ground state of the excited hydroxyl radical $(\mathrm{OH})$; there is also a poorly understood continuum throughout the visible waveband. A pure water SL spectrum will appear blue to bluish-white, but the presence of dissolved salts or other imare largely a coseismic occurrence, purities can appreciably alter the basic aqueous spectrum ${ }^{6}$, so that yellow or red may predominate.

SL has been generated in the laboratory with ultrasonic pressure amplitudes of 1-2 bar (0.1-0.2 MPa ${ }^{4}$, corresponding roughly to an energy density in the ambient fluid of $10-20$ erg $\mathrm{cm}^{-3}$. Whether SL is a viable mechanism for EQLs hinges on the question of whether it is reasonable that $P$ waves of subaudible frequency can supply this energy density in water.

The density of the kinetic energy, $e$ (per unit volume), induced in the transmission medium by one cycle of an advancing seismic wavefront is a standard result in seismology ${ }^{7}$ and is given by

$$
e=2 \pi^{2} \rho\left(A_{0} / \tau_{0}\right)^{2}
$$

where $\rho$ is the density of the medium and $A_{0}$ and $\tau_{0}$ are the displacement amplitude and period respectively of the seismic wave. Conservatively estimated values for $A_{0}$ of $1-10 \mathrm{~cm}$ and $\tau_{0}$ of 0.1-1.0 s may be obtained from the strong ground motion recordings of earthquakes. This yields $P$-wave energy densities in water of roughly $500-2,000$ erg cm$~^{-3}$ at $10-1 \mathrm{~Hz}$ and pressure differentials of $1.3-2.7$ bar. Thus, seismic $P$ waves are capable of supplying pressure changes and energy densities that exceed the laboratory values that induce SL. Within the water volume irradiated by $P$ waves, EQLs would arise as the integrated light flux from many SL cavitation bursts, all loosely synchronized by the $P$-wave dilational half-cycles.

In the laboratory, SL produces an illuminance of $\sim 10^{-8}$ lumen $\mathrm{cm}^{-2}$ (ref. 8 ), which is visible to the dark-adapted eye. Thus, to reproduce an illuminance equivalent to moonlight of $\sim 10^{-4}$ lumen $\mathrm{cm}^{-2}$, as reported for EQLs in Japan? $\sim 10^{4}$ ultrasonic SL bursts are required in the laboratory. For the much larger $P$-wave cavitation events (with bubble radius in the centimetre rather than micrometre range), the same illuminance could arise from a single event. To illuminate a landscape to moonlight brightness from at least several kilometres distance, $10^{2}-10^{4}$ individual SL $P$-wave bursts would be sufficient. A $P$-wave with a dilational half-cycle wavelength of $\sim 1 \mathrm{~km}$ is certainly cap-

\section{Correction}

IN the letter from M. Allard et al. (Nature 353, 610: 1991) "Tests for rodent polyphyly", the lettering on the figure, part $a$, was incorrect. The correct figure is shown below.
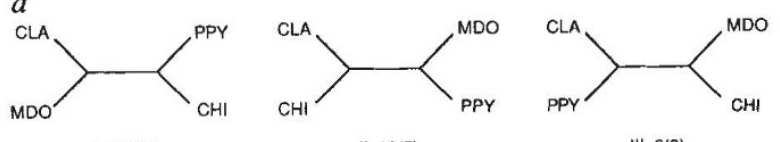

1 17(11) neither are they extremely rare. Selected characteristics relevant to the SL hypothesis that appear in the EQL literature are: (1) distinct blue to bluish-white EQLs reported from coastal Japan ${ }^{10}$ and Hawaii $^{2}$; (2) extensive EQLs from an onshore alluvial setting ${ }^{11}$; (3) numerous accounts of EQLs sighted offshore from California $^{1}$, Mexico $^{11}$ and other coastal zones; and (4) well-defined, blue and yellow spherical lights in tsunami wavecrests $^{12}$. (Luminescent organisms may be another source of luminescence in tsunami wavecrests ${ }^{13}$, but I do not believe that they would create welldefined spheres of light.)

Natural SL is perhaps not the only mechanism that produces EQLs, but it is able to explain a wide range of the existing reports. The hypothesis predicts that (1) EQLs should not be confined to the immediate fault rupture zone; (2) bodies of water must be present, although it is possible that saturated soil can sustain SL; (3) EQLs are essentially a coseismic phenomenon, which in the absence of strong foreshocks or aftershocks should not be observed before or more than several minutes after the earthquake; and (4) the EQL spectrum should contain a prominent hydroxyl peak at $310 \mathrm{~nm}$ (and for sea water ${ }^{6}$ a sodium peak at $589 \mathrm{~nm}$ ). Hence the SL-EQL hypothesis can be tested by means of spectrographic analysis, although obtaining an EQL spectrum will not be a trivial undertaking.

ARCH C. JOHNSTON

Center for Earthquake Research,

Memphis State University,

Memphis, Tennessee 38152, USA

1. Derr, J. Bull. seis. Soc. Am. 63, 2177-2187 (1973).

2. Lockner, D. A., Johnston, M. J. S. \& Byerlee, J. D. Nature 302, 28-33 (1983).

Brady, B. T. \& Rowell, G. A. Nature 321, 488-492 (1986)

4. Barber, B. P. \& Putterman, S. J. Nature 352, 318-320 (1991)

5. Sustick, K. S. Science 247, 1439-1445 (1990).

6. Suslick, K. S. \& Flint, E. B. J. Phys. Chem. 95, 1484 (1990)

Kasahara, K. Earthquake Mechanics (Cambridge University Press, 1981)

8. Waiton, A. J. \& Reynolds, G. T. Adv. Phys, 33, 595-660 (1984)

9. Musya, K. Bull. Earthquake Res. Inst. Tokyo Univ. 9, 214-215 (1931).

0. Terada, T. Bull. Earthquake Res. Inst. Tokyo Univ. 9, 225-255 (1931).

11. Fuller, M. L. U.S. Geol. Surv. Bult. 494, 120 (1912) Musya, K. Bull. Earthquake Res. Inst. Tokyo Univ. 10, 666-673 (1932).

13. Terada, T. Bull. Earthquake Res. Inst. Tokyo Univ. 1 25-35 (1934) able of spawning such an SL field.

Reports of EQLs for large nocturnal earthquakes are not ubiquitous, but 